\title{
On the Symmetric Algebra of a Module
}

\author{
Craig Huneke* \\ Department of Mathematics, University of Michigan, Ann Arbor, Michigan 48109 \\ Communicated by N. Jacobson \\ Received March 15, 1980
}

\section{INTRODUCTION}

The purpose of this article is to give a simple criterion in order for the symmetric algebra, $S_{R}(M)$, of an $R$-module $M$ to be a domain. Of course, we must assume $R$ is a domain. Before we state the main result let us introduce some notation.

If $A$ is a matrix with coefficients in a commutative ring $R$, by $I_{i}(A)$ we denote the ideal generated by the $t \times t$ minors of $A$. If $M$ is an $R$-module we denote the least number of generators of $M$ by $v(M, R)$. We recall a commutative Noetherian ring $R$ is said to satisfy Serre's property $S_{k}$ if

$$
\operatorname{depth} R_{p} \geqslant \min \left\{\operatorname{dim} R_{p}, k\right\}
$$

for all primes $p$ in $R$. If $I$ is an ideal in $R$, by grade $(I)$ we denote the length of a maximal $R$-sequence in $I$. We may now state the main result.

THEOREM 1.1. Let $R$ be a universally catenarian Noetherian domain satisfying $S_{m}$ and let $M$ be an $R$-module having a finite free resolution,

$$
0 \rightarrow R^{m} \stackrel{A}{\rightarrow} R^{n} \rightarrow M \rightarrow 0, \quad A=\left(a_{i j}\right) .
$$

Then the following are equivalent:

(1) $S_{R}(M)$ is a domain.

(2) $\operatorname{grade}\left(I_{i}(A)\right) \geqslant m+2-t$ for $1 \leqslant t \leqslant m$.

(3) $v\left(M_{p}, R_{p}\right) \leqslant n-m+\operatorname{grade}(p)-1$ for all non-zero primes $p$ in $R$.

If any (and hence all) of the above conditions hold, then $S_{R}(M)$ is a complete intersection in $R\left[T_{1}, \ldots, T_{n}\right]$. In particular, if $R$ is Cohen-Macaulay (respectively Gorenstein) then $S_{R}(M)$ is Cohen-Macaulay (respectively Gorenstein).

* Jr. Fellow, Michigan Society of Fellows. 
Several authors have investigated the case in which $S_{R}(I)$ is a domain if $I$ is an ideal. See $[6,8-10]$. The present author has used the idea of a $d$ sequence to provide a wide class of examples such that $S_{R}(I)$ is a domain. In this case, $\operatorname{gr}_{I}(R)$, the graded algebra of $I$, will be isomorphic to $S_{R / I}\left(I / I^{2}\right)$, and consequently is considerably easier to deal with. This author used this approach in [7] to prove results about the symmetric powers of prime ideals which are almost complete intersections, generic Pfaffians, or maximal minors of a generic $n \times(n+1)$ matrix. Also treated in [7] is the following well-known result (see [4] or [11]).

Proposition. Suppose $R$ is a commutative Noetherian domain and $M$ a finitely generated module, with resolution

$$
0 \rightarrow R \stackrel{\left(a_{1}, \ldots, a_{n}\right)}{\longrightarrow} R^{n} \rightarrow M \rightarrow 0 .
$$

If $\operatorname{grade}\left(a_{1}, \ldots, a_{n}\right) \geqslant 2$, then $S_{R}(M)$ is domain.

We will recover this result as an immediate corollary of Theorem 1.1. We will apply the theorem to several situations (these results may be found in Section 2). For instance, we show the following corollary, which we apply to primes defining varieties of the form $k\left[t^{n_{1}}, t^{n_{2}}, t^{n_{3}}\right]$, where $k$ is a field.

Corollary. Let $R$ be Cohen-Macaulay local domain and $p$ a height 2 prime generated by three elements, having projective dimesion 1 . Then $S(p)$ is a domain.

We now recall some facts about $S_{R}(M)$, the symmetric algebra of a module $M$. It is a non-negatively graded algebra. If $S^{n}(M)$ denotes its $n$th graded piece then

$$
S^{n}(M)=\underbrace{M \otimes \cdots \otimes M / N}_{n \text {-times }}
$$

where $N$ is the submodule of $M \otimes \cdots \otimes M$ generated by elements of the form

$$
\cdots \otimes m \otimes \cdots \otimes m^{\prime} \otimes \cdots-\cdots \otimes m^{\prime} \otimes \cdots \otimes m \otimes \cdots .
$$

If $M$ has the presentation

$$
R^{m} \stackrel{\left(a_{i j}\right)}{\longrightarrow} R^{n} \rightarrow M \rightarrow 0
$$

then $S_{R}(M)$ can be identified with $R\left[T_{1}, \ldots, T_{n}\right] / J$, where $J$ is the ideal generated by the $m$ linear forms $\sum_{i=1}^{n} a_{i j} T_{j}$. 
$S_{R}(M)$ has the following universal property: if $S$ is an $R$-algebra and $f: M \rightarrow S$ is an $R$-module homomorphism, then there exists a unique $f^{*}: S_{R}(M) \rightarrow S$ extending the identifications of $M$ as $S^{1}(M)$; i.e., the following commutes:

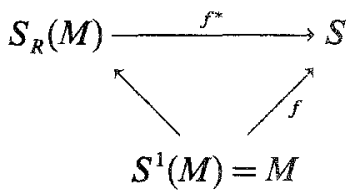

\section{The MAIN THEOREM}

We now begin the proof of Theorem 1.1. We will show that (2) implies (1), (1) implies (3), and (3) implies (2). So now assume (2).

Suppose grade $\left(I_{t}(A)\right) \geqslant m+2-t$ for $1 \leqslant t \leqslant m$. From the Introduction, we noted that

$$
S_{R}(M) \cong R\left[T_{1}, \ldots, T_{n}\right] / J,
$$

where $J$ is the ideal generated by the $m$ linear equations $\sum_{j=1}^{n} a_{i j} T_{j}$.

Since $\operatorname{grade}\left(I_{m}(A)\right) \geqslant m+2-m=2$ it is well known that $M$ must be torsion-free of rank $d=n-m$. By standard arguments we may imbed $M$ in $R^{d}$. (Let $K=$ fraction field of $R ; M$ imbeds in $M \otimes{ }_{R} K \cong K^{d}$. Take generators and clear denominators.)

Taking the symmetric algebras of this imbedding we obtain a map $f$,

$$
f: S_{R}(M) \rightarrow R\left[X_{1}, \ldots, X_{d}\right] .
$$

Denote by $Q$ the kernel of $f$ lifted back to $R\left[T_{1}, \ldots, T_{n}\right]$. Then $Q$ is a "generic point" for $S_{R}(M)$ in the following sense: $S_{R}(M)$ is a domain if and only if $Q=J$. For clearly if $Q=J$ then $S_{R}(M)$ is a domain. Conversely $S_{R}(M)$ a domain implies $f$ is an imbedding as then height $J=$ height $Q$ (invert a maximal minor) and so $Q=J$. The height $(Q)$ can be computed by the above remarks: $\operatorname{dim} S_{R}(M) / Q=\operatorname{dim} R\left[X_{1}, \ldots, X_{d}\right]=\operatorname{dim} R+d$. Hence height $Q=\operatorname{dim}\left(R\left[T_{1}, \ldots, T_{n}\right]\right)-(\operatorname{dim} R+d)=n-d=m$.

Now induct on $n=$ (the minimal number of generators of $M$ ) to show that under these conditions $S_{R}(M)$ is a domain. If $n=1$, then the conditions force $M$ to be torsion-free, $M \cong R$ and $S_{R}(M) \cong R[X]$ is a domain.

So assume $n>1$ and we have proved this half of the theorem for all $N$,

$$
0 \rightarrow S^{p} \rightarrow S^{k} \rightarrow N \rightarrow 0, \quad \text { where } k<n,
$$

and $S$ a universally catenarian Noetherian domain. 
Set $a=a_{i j}$ and consider $R_{a}$. Over $R_{a}$ we may change $A$ to the matrix

$$
\left(\begin{array}{cccc}
1 & 0 & \cdots & 0 \\
0 & & \\
\vdots & A^{\prime} \\
0 &
\end{array}\right)
$$

and clearly $\left(I_{t}\left(A^{\prime}\right)\right)_{a}=\left(I_{t+1}(A)\right)_{a}$. This gives an exact sequence

$$
0 \rightarrow R_{a}^{m-1} \stackrel{A^{\prime}}{\longrightarrow} R_{a}^{n-1} \rightarrow M_{a} \rightarrow 0 .
$$

Now $\operatorname{grade}\left(I_{t}\left(A^{\prime}\right)_{a}\right)=\operatorname{grade}\left(I_{t+1}(A)_{a}\right) \geqslant m+2-(t+1)=(m-1)+2-t$. Hence the assumptions hold for $A^{\prime}$ and we may assume $S_{R_{a}}\left(M_{a}\right)=\left(S_{R}(M)\right)_{a}$ is a domain. By the discussion above this implies $a^{n_{1}} Q \subseteq J$ for some $n_{1}$ and so there is $N \gg 0$ such that $I_{1}(A)^{N} Q \subseteq J$.

We claim this implies height $J=m$. For if $J \subseteq P$, a prime, and height $P<m$, then since $I_{1}(A)^{N} Q \subseteq P$ either $I_{1}(A) \subseteq P$ or $Q \subseteq P$ and so we see $J$ must have height $m$. But $J$ is generated by $m$ elements and $R\left[T_{1}, \ldots, T_{n}\right]$ satisfies $S_{m}$. Hence every associated prime of $J$ has grade at most $m$. But grade $\left(I_{1}(A)\right) \geqslant m+1$ and so $I_{1}(A)$ cannot consist of zero divisors modulo $J$. But $I_{1}(A)^{N} Q \subseteq J$ then forces $Q=J$ and so $S_{R}(M)$ is a domain.

Assume $S_{R}(M)$ is a domain. Induct on $n$ to show (3). We first show a special case of (3).

Lemma 1.1. Let $R$ be a Noetherian local domain satisfying $S_{k}$ and let $N$ be $n R$-module with a minimal resolution,

$$
0 \rightarrow R^{k} \stackrel{B}{\longrightarrow} R^{d} \rightarrow N \rightarrow 0 .
$$

If $S_{R}(N)$ is a domain, then $k \leqslant \operatorname{depth} R-1$.

Proof. By the argument above, if $S_{R}(N)$ is a domain, then $S_{R}(N)=$ $R\left[T_{1}, \ldots, T_{d}\right] / Q$, where $Q$ is prime and height $Q=k$. Also $Q$ is generated by $\sum_{j=1}^{d} b_{i j} T_{j}$. Hence $Q \subseteq I_{1}(B)$. But $Q \neq I_{1}(B)$ since clearly $b_{i j} \notin Q$.

Since the resolution is minimal, $I_{1}(B)$ is contained in $m$, the maximal ideal of $R$. In addition, since $R$ satisfies $S_{k}$, so does $R\left[T_{1}, \ldots, T_{d}\right]$ and so as $Q$ is a height $k$ ideal generated by $k$ elements, these elements form an $R\left[T_{1}, \ldots, T_{d}\right]-$ sequence. Since $Q$ is a prime contained in $m$, the grade of $m$ in $R\left\lfloor T_{1}, \ldots, T_{d}\right\rfloor$ must be at least $k+1$. Since the map from $R$ to $R\left[T_{1}, \ldots, T_{d}\right]$ is faithfully flat, the grade of $m$ in $R$ must be at least $k+1$ which concludes the proof of Lemma 1.1. 
We now show (3). Since $S_{R}(M)$ is a domain, $M$ is torsion-free and $\operatorname{rank}(M)=\operatorname{rank}\left(M_{p}\right)=n-m$ is well defined. Since $S_{R_{p}}\left(M_{p}\right)=\left(S_{R}(M)\right)_{W}$. where $W=R-p$, to show (3) it is enough to assume $R$ is local with maximal ideal $m$ and to show

$$
v(M, R) \leqslant n-m+\operatorname{depth}(R)-1 .
$$

We may choose $n=v(M, R)$, and so it is enough to show

$$
m \leqslant \operatorname{depth}(R)-1 .
$$

However, this is precisely the statement of Lemma 1.1.

We now show (3) implies (2). Observe that if $p$ is a prime in $R$, then $I_{t}(A)$ is not contained in $p$ if and only if $v\left(M_{p}, R_{p}\right) \leqslant n-t$, as in this case we may transform $A$ (over $R_{p}$ ) into

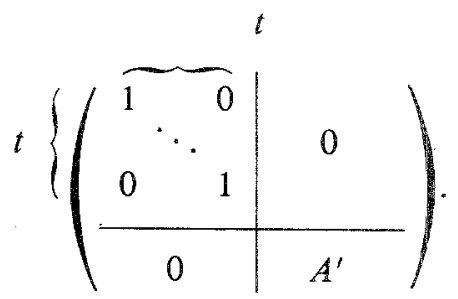

Now suppose (3) holds, yet grade $\left(I_{t}(A)\right) \leqslant m+1-t$. Hence there is a prime $p$ containing $I_{t}(A)$ whose grade is at most $m+1-t$. From the observation above, we deduce $v\left(M_{p}, R_{p}\right)>n-t$. However, by (3), $v\left(M_{p}, R_{p}\right) \leqslant$ $n-m+(m+1-t)-1=n-t$. This contradiction completes this part of the proof.

To complete the proof we observe that if $S_{R}(M)$ is a domain then as has been observed, the prime $Q$ defining it has height $m$ and is generated by $m$ elements. Since $R$ satisfies $S_{m}$, these elements must form an $R\left[T_{1}, \ldots, T_{n}\right]$ sequence.

COROLlary 1.1. Suppose $R$ is a Cohen-Macaulay domain and $M a$ module as in Theorem 1.1. If $S_{R}(M)$ is a domain then

$$
v(R, M) \leqslant \operatorname{rank} M+\operatorname{dim} R-1 .
$$

Proof. We use the result of Forster [1].

Lemma $1.2[1]$. Let $R$ be a Noetherian ring, and $M$ an $R$-module. Then

$$
v(R, M) \leqslant \sup \left\{v\left(R_{p}, M_{p}\right)+\operatorname{dim} R / p\right\} .
$$


We apply this together with (3) of Theorem 1.1.

$$
v\left(R_{p}, M_{p}\right) \leqslant \operatorname{rank} M+\operatorname{height}(p)-1 .
$$

Hence,

$$
\sup \left\{\operatorname{dim} R / p+v\left(R_{p}, M_{p}\right)\right\} \leqslant \operatorname{dim} R+\operatorname{rank} M-1 .
$$

Corollary 1.2. Let $R$ be a Cohen-Macaulay local domain and $p$ a height 2 prime having projective dimension 1 and having three generators. Then $S_{R}(p)$ is a domain.

Proof. From (3) of Theorem 1.1. we need only check that $v\left(p_{q}, R_{q}\right) \leqslant$ height $(q)$. Since $p$ has only three generators we only needs to check this at $p$; however, $R_{p}$ is regular so $v\left(p_{p}, R_{p}\right)=2$.

\section{Applications}

In [4], Hochster studied rings of the form $R\left[T_{1}, \ldots, T_{n}\right]$ modulo a linear form, $a_{1} T_{1}+\cdots+a_{n} T_{n}$. He called these generic grade reductions. We note that one simple result follows immediately from Theorem 1.1.

Proposition 2.1. Suppose $R$ is a commutative Noetherian domain and $M$ is defined to be a cokernel of the map,

$$
0 \rightarrow R \stackrel{\left(a_{1}, \ldots, a_{n}\right)}{\longrightarrow} R^{n} .
$$

If $\operatorname{grade}\left(a_{1}, \ldots, a_{n}\right)$ is at least 2, then $S_{R}(M)=R\left[T_{1}, \ldots, T_{n}\right] /\left(a_{1} T_{1}+\cdots+a_{n} T_{n}\right)$ is a domain.

Proof. Since $R$ is a domain, $R$ satisfies $S_{1}$. We now apply (2) of Theorem 1.1.

Proposition 2.2. Let $k$ be a field and let $p$ be the prime in $k[X, Y, Z]$ defining the variety $k\left[t^{n_{1}}, t^{n_{2}}, t^{n_{3}}\right]$. Set $R=k[X, Y, Z]$. Then $S_{R}(p)$ is a Gorenstein domain.

Proof. Herzog[2] has shown that $p$ is generated by at most three elements. Since $R$ is regular and $R / p$ is Cohen-Macaulay with height $(p)=2$, we see the projective dimension of $p$ is 1 . Corollary 1.2 shows $S_{R}(p)$ is a domain and Theorem 1.1 shows $S_{R}(p)$ is Gorenstein since $R$ is.

Proposition 2.3 [3]. Let $k$ be a field, and fix numbers $0<t_{1}<$ $t_{2}<\cdots<t_{n}=r$. Let $X_{i}$ be a generic $t_{i} \times t_{i+1}$ matrix and set 
$X=X_{1} \ldots X_{n-1}$. Let $T$ be a generic $r \times 1$ matrix. Set $R=k\left[X_{1}, \ldots, X_{n-1}, T\right]$ by which we mean $k$ with the entries of the matrices adjoined. Let $J$ be the ideal generated by the entries of XT. Then $J$ is prime and is generated by an R-sequence.

Proof. Set $S=k\left[X_{1}, \ldots, X_{n-1}\right]$ and $t=t_{1}$. The matrix $X$ defines a module $M$ by the exact sequence,

$$
0 \rightarrow S^{t} \stackrel{x}{\longrightarrow} S^{r} \rightarrow M \rightarrow 0 .
$$

Further, $S_{S}(M)$ is $R / J$. Hence, provided height $\left(I_{i}(X)\right)$ is at least $t+2-i$, Theorem 1.1 will give the required conclusion. However, the nilradical of $I_{i}(X)$ contains $I_{i}\left(X_{1}\right) \cdots I_{i}\left(X_{n-1}\right)$, and it is well known (see, for example, [3]) that

$$
\operatorname{grade}\left(I_{i}\left(X_{j}\right)\right)=\left(t_{j}-i+1\right)\left(t_{j+1}-i+1\right) .
$$

Since $i$ is at most $t$ and $t$ is strictly less than $t_{2}$ this is evidently at least $t+2-i$.

\section{ACKNOWLEDGMENT}

The author would like to thank R. Cowsik for many insightful comments and in particular for the observation that $S_{R}(M)$ a domain implies it is a complete intersection.

\section{REFERENCES}

1. O. Forster, Über dic Anzahl der Erzeugenden eines Ideals in einem Noetherschen Ringe, Math. Z. 84 (1964), 80-87.

2. J. Herzog, Generators and relations of Abelian semigroups and semigroup rings, Manuscripta Math. 3 (1970), 175-193.

3. J. EAGON AND M. Hochster, Cohen-Macaulay rings and the generic perfection of determinantal loci, Amer. J. Math. 93 (1971), 1020-1058.

4. M. Hochster, Properties of Noetherian rings stable under general grade reduction, Arch. Math. 24 (1975).

5. C. HUNEKE, The theory of $d$-sequences and powers of ideals, Advances in Math.; in press.

6. C. HUNEKE, On the symmetric and Rees algebras of an ideal generated by a $d$-sequence, J. Algebra 62 (1980), 268-275.

7. C. HUNEKE, Symbolic powers of prime ideals and special graded algebras, to appear.

8. A. Micali, Sur les algèbres symetrique et de Rees d'un ideal, Ann. Inst. Fourier.

9. A. Micali, P. Salmon, and P. Samuel, "Integrité et factoiralité des algebras symétriques," Atas do IV Coloquio Brasileiro le Mathematica, Săo Paulo, 1965.

10. P. SAlmon, "Sulle algebre simmetricie e di Rees di un ideale," Edizioni Scientifiche, Genoa, 1964.

11. P. SamurL, Anneaux gradués factorials et modules réflexifs. Bull. Soc. Math. France 92 (1964), 237-249. 